\title{
Desarrollo del emprendimiento en los estudiantes de ingeniería mediante aprendizaje basado en proyectos
}

\author{
Development of Entrepreneurship in Engineering Students Through Learning Based on Projects \\ Ma. Antonia Moreno- Aguilar ${ }^{a}$, Guillermina Sánchez- López ${ }^{b}$, Ramón Beltrán Martínez $^{c}$
}

\begin{abstract}
:
The vertiginous advance of technological development spreads to all the population levels where new "needs" are generated in many aspects like physical, mental, social and the familiar one, which must be satisfied at the rhythm of these advances. Owing to the previous reasons mentioned it has been imperious the update of the methodology implemented for the education of engineering students, all to promote in these, the development of two main characteristics: innovation and entrepreneurship.

What promotes in the future engineer the development of the abilities, skills, aptitudes, attitudes and knowledge necessary to achieve the transformation of the environment where they operate in the workplace.

A learning-teaching strategy that allows the development of competences in engineering students, is the Project-Based Learning, because of this it is proposed to implement it in the subject Development of Bioenergy projects of the Universidad Technologic de Puebla, using as a statistical tool the Design of experiments, with the purpose of establishing the optimal conditions for the improvement of the quality in the development of the projects realized by the student.
\end{abstract}

Key words:

Entrepreneurship, projects, production

\section{Resumen:}

El vertiginoso avance del desarrollo tecnológico contagia a todos los niveles de la población, donde se generan nuevas "necesidades" tanto físicas, mentales, sociales, familiares que al ritmo de esos avances deben ser satisfechas. Debido a lo anterior se hace imperiosa la actualización de la metodología implementada para la formación de los estudiantes de ingeniería siendo necesario promover en estos, el desarrollo de dos características principales: la innovación y el emprendimiento.

Lo que promueve en el futuro ingeniero el desarrollo de las habilidades, destrezas, aptitudes, actitudes y conocimientos necesarios para lograr la transformación del entorno donde se desenvuelve en el ámbito laboral.

Una estrategia aprendizaje-enseñanza que permite el desarrollo de las competencias en los estudiantes de ingeniería, lo representa el Aprendizaje Basado en Proyectos, debido a esto es que se plantea implementarlo en la materia Desarrollo de proyectos de Bioenergía de la Universidad Tecnológica de Puebla, utilizando como herramienta estadística el Diseño de experimentos, con la finalidad de establecer las condiciones óptimas para la mejora de la calidad en el desarrollo de los proyectos realizados por el estudiante

\section{Palabras claves:}

Emprendimiento, proyectos, producción

\section{Introducción}

El ingeniero, profesionalmente hablando, debe actuar, proponer y decidirse, haciendo frente a todo tipo de retos que se le presenten. 1
Un emprendedor se define como "el que organiza, administra y asume el riesgo de un negocio, empresa o nueva tecnología". Para lograr el cumplimiento de la anterior definición son necesarias: la investigación, el

a Autor de Correspondencia, Benemérita Universidad Autónoma de Puebla, ORCID: 0000-0001-5866-9362, Email: ma.moreno.aguilar@gmail.com

b Instituto Tecnológico de Puebla. Email: guille.sanlopez@gmail.com

c Universidad Tecnológica de Puebla, División Energías Renovables, Email: ramon.beltran @utpuebla.edu.mx 
análisis, el desarrollo, el diseño y la evaluación de nuevas tecnologías. 2

Por tanto, para poder desarrollar el emprendimiento en el ingeniero es necesario promover la innovación en la los conceptos de innovación y creatividad que sirven como valores fundamentales en este proceso. 3

Hasta hace unos años la formación del ingeniero estaba encaminada únicamente a tener un cierto volumen de conocimientos técnicos, actualmente estos profesionistas deben identificar con qué recursos cuenta y como transformarlos, ubicar donde se encuentra y establecer la ruta adecuada para llegar a donde planea, minimizar sus debilidades y potencializar sus fortalezas para aprovechar las oportunidades, eliminando las amenazas.

Debe también en su desarrollo profesional ser capaz de actuar independientemente, sin presiones, cambiando paradigmas, transformando sus ideas, desarrollando nuevos talentos, aprendiendo y desaprendiendo, confiando en si mismo y en sus capacidades, aprovechando las oportunidades de mejora continua que se les presente, es decir buscando la rentabilidad económica de ese conocimiento.

Siendo justamente lo anterior lo que hace necesaria la educación en el emprendimiento, ya que es posible "enseñar "a emprender.

En la mente del emprendedor el futuro no existe, lo imagina y lo construye, por lo que se contribuye a la resolución de los problemas presentes y futuros de la sociedad. 4

De manera que actualmente es necesario encontrar las técnicas adecuadas mediante las cuales pueda capacitarse a los estudiantes en emprendimiento, tratando de lograr la conceptualización del "espíritu emprendedor" como un enfoque multidisciplinario que tiene como finalidad, la creación y la innovación frente a la incertidumbre y los recursos limitados.

Con respecto a lo anterior, a nivel superior, en los perfiles de En el programa de estudios de cada asignatura se hace mención de cómo ésta contribuye al perfil de egreso apoyando a los estudiantes a desarrollar estas competencias, por lo que es se hace necesario transmitirles no sólo el conocimiento propio del curso, sino el interés de plantear e investigar problemas reales, cuyas soluciones sean benéficas a su entorno, que es la base del emprendimiento. investigación, con lo cual, se provoca un liderazgo de los ingenieros en el ámbito empresarial que ayuda a desarrollar

El lograr que el aprendizaje sea significativo para el estudiante es de suma importancia en el desarrollo de todo curso, para esto, existen diferentes modelos educativos donde se establecen estrategias enseñanzaaprendizaje, para alcanzar este fin.

Con la finalidad de lograr que los ingenieros formados en la Universidad Tecnológica de Puebla UTP alcance una formación completa, tanto teórica como práctica, y tomando como base su perfil de egreso, donde uno de los propósitos que se plantean es el desarrollo de conocimientos, habilidades, destrezas, aptitudes, actitudes y valores, que permitan al egresado desarrollarse en el terreno laboral, se hace necesario fomentar el emprendimiento, por lo que se propone implementar una adecuación al aprendizaje basado en proyectos como el eje rector de la asignatura "diseño de proyectos de bioenergía", con la intención de desarrollar las competencias que le permitan originar una idea, plasmarla, planearla y concretarla en físico, impulsando tanto la creatividad como el espíritu emprendedor.

Si bien el aprendizaje basado en proyectos permite que el estudiante desarrolle sus habilidades destrezas y capacidades, aplicando los conocimientos apropiados; es necesario realizar una metodología correcta que le permita obtener observaciones precisas y sobre todo que pueda tomar decisiones correctas y precisas.

Estas decisiones pueden ayudar a que una empresa pueda salir adelante, o que la producción de algún bien o servicio sea redituable, pero para determinar lo anterior, en todo proceso es necesario analizar todas las variables que se encuentra presentes y que causan modificación en los resultados.

Todo lo anterior resulta ser importante en la formación del estudiante ya que se le está preparando para formar parte del terreno laboral o bien continuar con una preparación académica más especializada.

En este contexto, este documento da una propuesta de estrategia aprendizaje-enseñanza con la finalidad de mejorar la práctica educativa desde una perspectiva de emprendimiento que promueva el aprendizaje basado en

proyectos en la Universidad Tecnológica de Puebla, impulsando el pensamiento y actuar creativo, generando la capacidad de tomar decisiones acertadas. 


\section{Materiales y métodos}

Un modelo educativo, es un planteamiento surgido de la necesidad de simbolizar y representar la tarea de la enseñanza- aprendizaje que el docente realiza, determinando el grado de conocimiento generado en el estudiante para transformar su práctica según los resultados obtenidos. 5

Esta función, se describe como que un modelo de enseñanza adopta la representación mental más valiosa y apropiada para mejorar tanto el conocimiento práctico como el teórico. 6

Existen diversos modelos educativos los cuales plantean la enseñanza de diversas formas, algunas centradas en:

- La transmisión de la información

- Los valores

- En el estímulo respuesta

- La actividad de los alumnos

- En los procesos de aplicación

Vygotsky establece el constructivismo, como un modelo en el que el estudiante sea el que mediante procesos cognitivos vaya construyendo sus saberes, es decir, darles un sentido, con la dirección del docente, para que, logre un aprendizaje significativo, en este modelo constructivista el aprendizaje por descubrimiento juega un papel importante ya que al encontrar varias soluciones para un mismo problema, promueve en el estudiante el interés por investigar qué es lo correcto, con esto, debe lograrse que los nuevos conocimientos entren a la zona de desarrollo próximo, donde el individuo es capaz de recordar y aplicar lo aprendido.

Por otro lado, el relacionar en el estudiante el aprendizaje mecánico con el aprendizaje significativo, en el primero no se necesitan conocimientos previos del tema en cuestión, en el significativo en cambio, se relacionan todo los conceptos estudiados y se realiza la aplicación de ellos, es decir es un "continuum" debiendo unirse ambos aprendizajes, en el trabajo con el estudiante, es necesario promover que el conocimiento nuevo se una con el conocimiento previo para lograr ese tan proclamado aprendizaje significativo, autores propone que se implemente con los estudiantes materiales que ellos puedan relacionar dando un significado lógico, que le permita relacionarlo con las ideas que tiene en su estructura cognitiva.7

Si el significado que se adquiere es un contenido cognoscitivo nuevo, diferenciado e idiosincrático entonces se convierte como Ausubel decía en un significado psicológico, el cual requiere de contenidos teóricos suficientes en el estudiante para poder llegar a ser aplicado, lo cual en teoría es excelente, el problema se establece en el momento en el que el docente pierde el control del grupo y no logra aterrizar las ideas de los estudiantes, al no dirigir correctamente lo investigado y se convierte la clase en un procedimiento, en un método para hacer alguna cosa y se deja a un lado el enfatizar los conocimientos, los cuales deben ser la base de toda enseñanza. 7,8

Por esta razón evaluar al estudiante con exámenes estandarizados, o bien, impartir las asignaturas de forma expositiva, no dará prueba de la apropiación de conocimientos que tengan, en el mejor de los casos saben resolver problemas, pero no saben que significan los resultados que están obteniendo; esta situación hace imperativa la búsqueda de planeaciones efectivas que permitan construir el conocimiento aprendiendo $y$ aplicando conceptos teóricos.

No basta que el material sea significativo, debe existir también en el estudiante la inquietud por interpretar los resultados obtenidos, proceso en el cual el docente es el guía del alumno, aplicando las estrategias pertinentes para ello, motivando a que se realice la asimilación cognitiva requerida, de manera que se logre alcanzar el aprendizaje combinatorio en el cual hay una transferencia efectiva del aprendizaje. 7

Como parte importante de los modelos de enseñanza está el aprendizaje basado en proyectos, (ABP), el cual es un recurso que es recomendado por algunos investigadores, por ofrecer más ventajas que desventajas. 9

El utilizar un aprendizaje con base a proyectos obliga a los estudiantes a plantearse preguntas tales como: ¿Cuál es mi problema? ¿Necesito datos? ¿Cuáles? ¿Cómo puedo obtenerlos? ¿Qué significa este resultado en la práctica? 10

Así las ventajas de usar $A B P$ como una estrategia a considerar por parte del docente, son de acuerdo a diversos autores:

- Logran aprender de los errores cometidos

- Como resultado del tiempo empleado se obtiene un producto tangible

- Conforme se va avanzando se producen ideas novedosas y eficaces, para solucionar las problemáticas que se presentan, ante lo cual los estudiantes aplican un trabajo colaborativo necesario para la implementación de estas mejoras encontradas.

- El estudiante es protagonista de su aprendizaje, acentuándose su papel autónomo y activo. 
- El rol del docente es apoyar, recomendar, analizar y dar seguimiento del trabajo a realizar.

- Se inicia de una situación-problema que es el eje motivacional del trabajo de los estudiantes.

- Permite contextualizar a la asignatura en cuestión y hacerla más relevantes.

- Se aprende a identificar y comprender características de los datos reales (variabilidad precisión, fiabilidad, posibilidad de medición y sesgo).

- Desarrolla y promueve empatía entre los participantes.

- Promueve el trabajo disciplinar.

- Promueve la capacidad de investigación tanto grupal como individual.

- Provee de una herramienta y una metodología para aprender cosas nuevas de manera eficaz.

- Fomenta la responsabilidad de cada integrante.

- Favorece la toma de decisión.

- Favorece la relación; estudiante- entorno, donde él es capaz de determinar la responsabilidad como partícipe de la mejora de su entorno. 9

El modelo didáctico que más se adecúa a las necesidades actuales es el modelo alternativo en el cual se emplean diferentes estrategias metodológicas con la finalidad de trabajar por competencias, lo cual es necesario tanto a nivel superior como medio superior. 5 .

\section{Metodología}

La presente investigación se realiza bajo un enfoque cuantitativo, mediante un proceso deductivo que plantea inicialmente analizar el efecto que provoca en el estudiante la implementación del aprendizaje basado en proyectos cono eje rector del programa de estudios de una asignatura y la forma de mejorarlo y reforzarlo.

Las Fuentes utilizadas para el proyecto se fundamentaron en las siguientes fases:

- Se realizó una encuesta a egresados de la Ingeniería en energías renovables de la UTP obteniéndose que ningún encuestado ha creado su propia micro empresa, por lo que con la finalidad de coadyuvar al emprendimiento.

- Se establecen como objetivos a alcanzar, la implementación del aprendizaje basado en proyectos en la materia de Diseño de proyectos de bioenergía, con la finalidad de inducir el emprendimiento en los estudiantes.
- El presente trabajo es resultado de tres implementaciones en la misma materia en distintos cursos cuatrimestrales.

- En cada nueva implementación se van determinando y ajustando variables, con las experiencias obtenidas, lo cual permite establecer planes de acción.

- Se propone la implementación del diseño de experimentos con la finalidad de tener más orden y capacidad de predicción a largo plazo.

- Para esto la implementación se apoya en el marco teórico, abordado en el presente trabajo, el cual es el producto de la investigación de diferentes fuentes acerca del ABP.

\section{Implementación}

Este trabajo es el resultado de haber aplicado el aprendizaje basado en proyectos, en la asignatura "Diseño de proyectos de bioenergía" en tres ocasiones en diferentes cuatrimestres, teniendo como finalidad el lograr el desarrollo de la competencia que el programa de estudios marca.

Tras los resultados de la primera implementación, se observa la dificultad que al término del proyecto el estudiante tiene para concluir y presentar soluciones innovadoras como resultado de su experimentación. Por lo que se propone como mejora para la siguiente aplicación que las variables que deben tomarse en cuenta son: la planeación de la asignatura, el seguimiento al proyecto, el aprendizaje alcanzado por los estudiantes, la retroalimentación oportuna y la evaluación.

En la segunda aplicación de la metodología se cuidaron las variables antes mencionadas, encontrando que en las fases de la implementación del aprendizaje basado en proyectos que son inicio, desarrollo y final, es muy importante la guía del docente para alcanzar conclusiones efectivas en los tiempos establecidos.

Apareciendo por tanto una nueva variable a cuidar: Manejo de tiempos

Al término de esta segunda aplicación se propone un replanteamiento de la metodología con la finalidad de evaluar y por tanto mejorar lo que se había obtenido,

Para esto, retomamos perfil de egreso, objetivo de aprendizaje y competencia a desarrollar de la asignatura en cuestión. 
En el perfil de egreso de la ingeniería en energías renovables de la UTP, se plantea que el estudiante será capaz de formular, evaluar, integrar y dirigir proyectos de energías renovables mediante diagnósticos energéticos y estudios especializados de los recursos naturales del entorno para contribuir al desarrollo sustentable.

En tanto que la materia "Diseño de proyectos de bioenergía”, donde se aplicó la metodología tiene como objetivo de aprendizaje:

"El alumno diseñará sistemas de generación de biocombustibles a través de la transformación de la biomasa mediante software especializado para la producción de energías alternas y contribuir al desarrollo sustentable de la región y del mundo".

La competencia a desarrollar en la asignatura mencionada es: "Desarrollar sistemas de energías renovables mediante el diseño de soluciones innovadoras, administrando el capital humano, recursos materiales y energéticos para mejorar la competitividad de la empresa y contribuir al desarrollo sustentable de la región".

Siendo lo anterior lo que inspira la búsqueda de nuevas alternativas de mejora.

Es por tanto necesario desde cada una de las asignaturas que forman la currícula escolar de la universidad "aportar" a ese perfil de egreso

En la tercera aplicación se propone hacer uso del diseño de experimentos como una herramienta estadística que fortalezca el desarrollo y seguimiento del proyecto hacia el emprendimiento.

En este ámbito lo que se propone enfatizar en el estudiante es el emprendimiento, es decir, ¿cómo puedo hacer que mi proyecto abarque más? ¿Cuáles son las perspectivas? ¿Qué variables deben controlarse? ¿Cómo influyen a mi producto las variables que no se han controlado?

Como se sabe las fases del aprendizaje basado en proyectos son tres: inicial, en desarrollo y final.

La fase inicial implica la planeación y presentación del docente con la finalidad de dejar en claro, el tópico, el proceso a realizar por los estudiantes, las metas parciales y finales que deberán acreditar al término. Así como también establecer la fecha de las sesiones presenciales donde se definirán los procedimientos para desarrollar un proyecto complejo, la forma mediante la cual se adquirirán los conocimientos necesarios en cada etapa de este desarrollo.

\section{Conformación de los equipos.}

Es importante resaltar justamente que al irse desarrollando un proyecto surge en los estudiantes la necesidad de investigar conceptos nuevos que en su momento deben aplicar en la resolución de la problemática planteada, estos conocimientos no solo se refieren a conceptos teóricos, sino también al desarrollo de habilidades, destrezas, actitudes y aptitudes.

En el grupo de $10^{\circ}$ cuatrimestre se formaron 4 equipos para realizar el trabajo colaborativamente.

Como el programa de la asignatura lo plantea, los temas a estudiar a lo largo del cuatrimestre son: Biomasa, biogás y producción de hidrógeno.

Por lo que al inicio se les plantearon tres problemas a resolver a lo largo del cuatrimestre.

- Eficiencia en un biodigestor

- Mejora en la producción de hidrógeno

- Composta \& lombricomposta

La planeación de la sesión de clase es vital en el desarrollo de esta estrategia de aprendizaje- enseñanza, ya que el docente debe tomar en cuenta las situaciones que en dado caso puedan presentarse en los grupos donde se está trabajando.

El procedimiento que se utilizó con ellos fue el siguiente
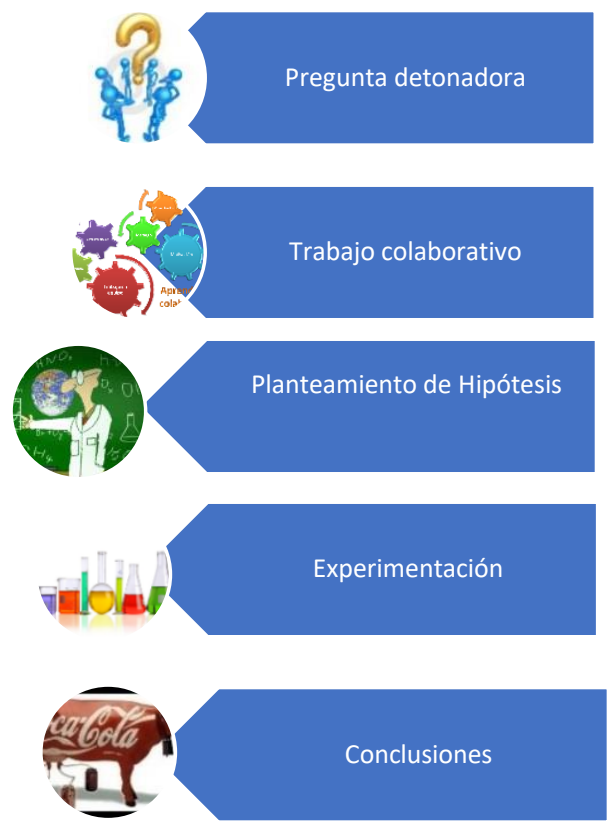

Figura 1. Diagrama de metodología 
La fase de desarrollo se realiza como se mencionó antes, empleando como herramienta estadística el diseño de experimentos con la finalidad de establecer las condiciones óptimas para la mejora de la calidad en el desarrollo de los proyectos realizados por el estudiante

Al aplicar el diseño de experimentos los estudiantes establecieron como condiciones del proceso eficiencia del biodigestor, las mostradas en el diagrama 2.

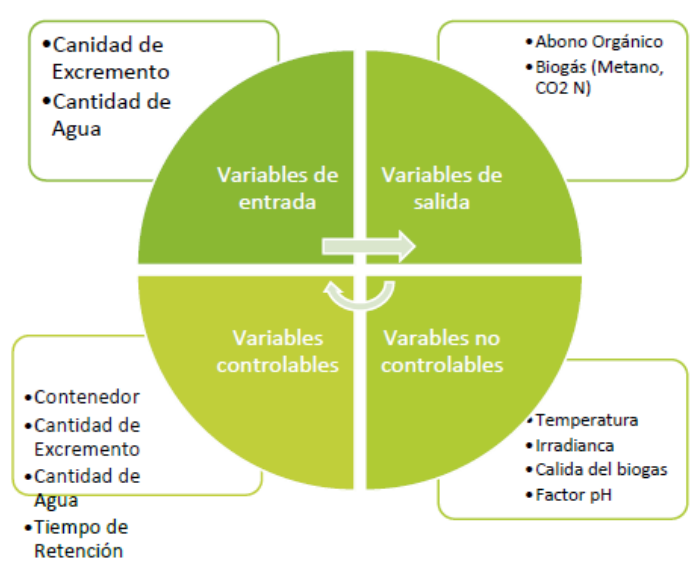

Figura 2. Proceso del biodigestor

Como se observa en el diagrama anterior, en la primera parte, el uso del diseño de experimentos permite establecer de una forma clara las variables que se encuentran presentes en el prototipo y cómo se relacionan entre sí, de manera que se determine durante el proceso quienes se vuelven controlables, quienes incontrolables y quienes serán las variables de salida, las cuales son las que finalmente permiten determinar la eficiencia del proceso.

EI DOE se utiliza para identificar las condiciones del proceso y los componentes del producto que afectan la calidad, para luego determinar la configuración de factores que optimiza los resultados.

En el caso de la comparación entre biodigestores, al realizar las mediciones correspondientes, se observa que es necesario hacer adecuaciones para mejorarlos, por lo que se plantean diversas adecuaciones con base en las variables estudiadas, para eficientar los procesos. (Imagen 1y 2)
En esta parte es importante que el estudiante observe la utilidad del diagrama para determinar los pasos que ha de seguir hasta lograr que su prototipo sea más eficiente.

Por otro lado, este tratamiento permite observar la repetibilidad y la replicabilidad entre las diferentes unidades experimentales, recordando que se les denomina así a los prototipos realizados por los diferentes equipos, por otra parte, replicabilidad se refiere a que bajo condiciones similares se fabrica otro prototipo, en tanto que la repetibilidad implica las mediciones en un mismo prototipo.

\begin{tabular}{|c|c|c|c|c|}
\hline Repetibilidad & \multicolumn{4}{|c|}{ Replicabilidad } \\
\hline Variables & $\begin{array}{c}\text { Prototipo } \\
1\end{array}$ & $\begin{array}{c}\text { Prototipo } \\
2\end{array}$ & $\begin{array}{c}\text { Prototipo } \\
3\end{array}$ & $\begin{array}{c}\text { Prototipo } \\
4\end{array}$ \\
\hline Estiércol & 2 & 1 & 5 & 2 \\
\hline Agua (I) & 2 & 3 & 5 & 2 \\
\hline Humedad (\%) & 80 & 65 & 90 & 85 \\
\hline $\mathrm{TI}\left({ }^{\circ} \mathrm{C}\right)$ & 19 & 18 & 18 & 17 \\
\hline TF. & 25 & 15 & 23 & 25 \\
\hline Olor & medio & medio & medio & bajo \\
\hline
\end{tabular}

Tabla 1. Mediciones del proceso

En la tabla 1 podemos observar tanto la replicabilidad como la repetibilidad del proceso correspondiente a la eficiencia de los biodigestores cabe hacer mención que en la tabla se muestran los resultados obtenidos por los 4 equipos diferentes

El diseño de experimentos (DoE), ayuda a investigar los efectos de las variables de entrada, sobre una variable de salida, al mismo tiempo. Estos experimentos consisten en una serie de corridas o pruebas, en las que se realizan cambios intencionales en las variables de entrada. En cada corrida se recolectan datos.
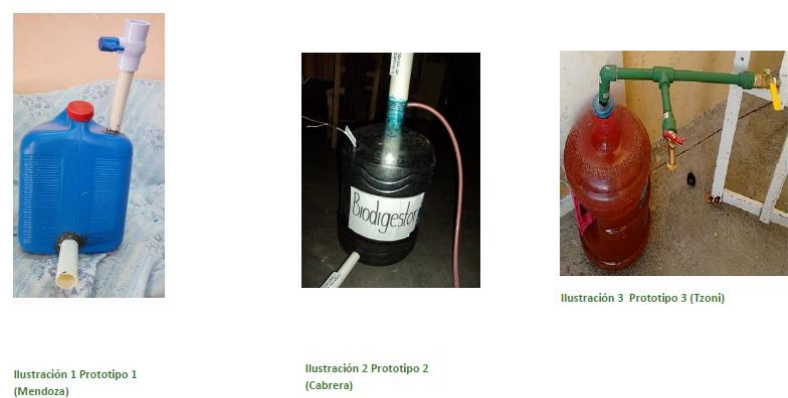

Figura 3. Biodigestor 


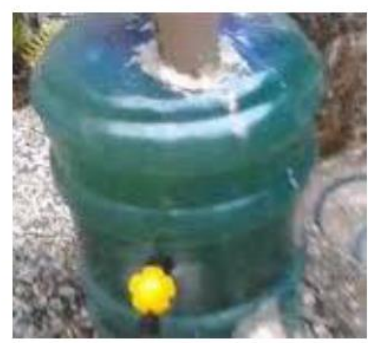

Figura 4. Biodigestor

Las imágenes anteriores muestran algunos de los prototipos realizados por los equipos, las mediciones obtenidas son mostradas en la tabla 1 , en la cual es posible observar los logros que a lo largo de su proyecto tuvieron los estudiantes.

Resultados y Discusión

En la fase de conclusión del aprendizaje basado en proyectos una parte muy importante es el seguimiento constante y continuo al estudiante, la tabla de resultados obtenidos no solo se puede interpretar como la eficiencia de un determinado prototipo, sino también como el desempeño de los estudiantes integrantes de un equipo determinado, lo cual puede ser mejor apreciado en las gráficas 1 y 2 que a continuación se presentan.

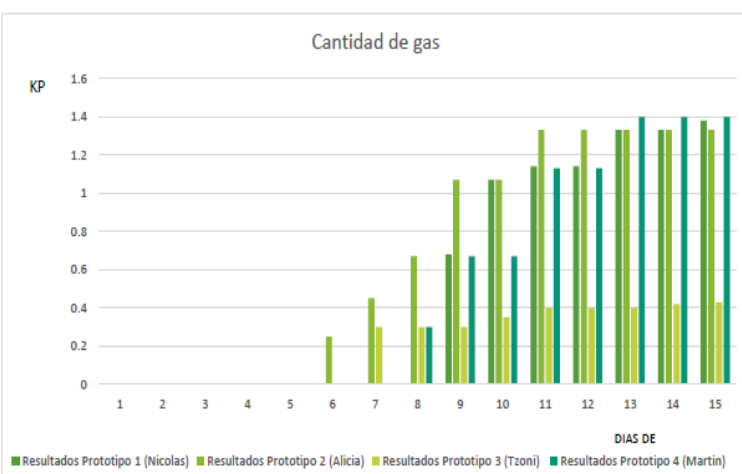

Gráfica 1

En la gráfica 1, se tiene que el equipo 2 obtuvo las mediciones más bajas en la elaboración del biodigestor

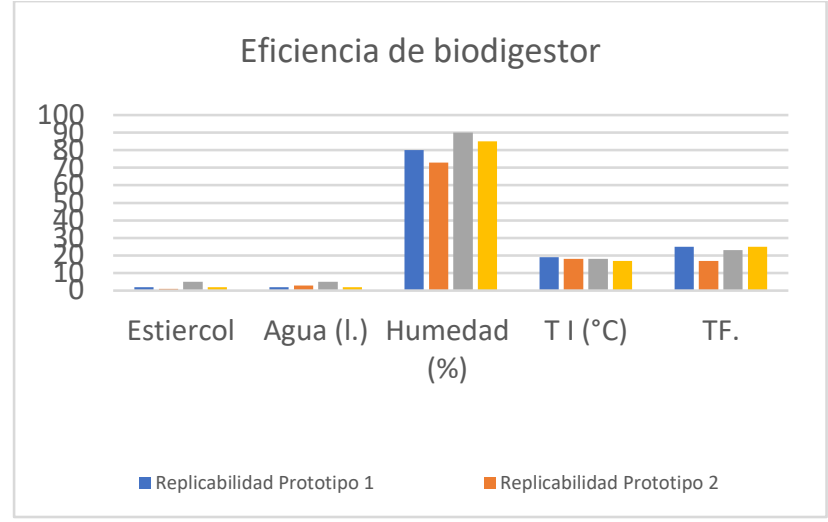

Gráfica 2

En la segunda ronda de mediciones y después de algunas adecuaciones (gráfica 2), el equipo 2 mejora su rendimiento, pero aún es más bajo su desempeño en comparación con los otros equipos.

Con los resultados obtenidos en este proyecto, se plantea por parte del docente realizar un seguimiento más cercano con los estudiantes del equipo 2, de manera que puedan detectarse el tipo de deficiencias que estos alumnos presentan, después de analizar e investigar, se observó que los estudiantes habían tenido un bajo desempeño a lo largo de su carrera, por lo que en los siguientes proyectos se trabajó más de cerca en todas las fases de sus trabajos, es decir, en la investigación, planteamiento, medición, conclusiones.

El aprendizaje basado en proyectos implementado en las ingenierías es una oportunidad para detectar las deficiencias y necesidades del estudiante en la aplicación real de sus conocimientos, el proyecto incluye la entrega de un reporte donde los equipos deben redactar tanto su investigación documental como los resultados obtenidos, detallando el modelo matemático que las mediciones presentan, con la finalidad de realizar el dimensionamiento adecuado que permita establecer la presentación de un proyecto a mayor escala.

Es importante resaltar que el reporte mencionado incluye una investigación de las instancias donde se pueden solicitar recursos económicos para poner en práctica si es factible su prototipo en la formación de una empresa, esta investigación detalla tanto los montos como los requisitos para obtenerlos.

En el desarrollo de proyectos es básico que el estudiante tenga el conocimiento del impacto de su propuesta, con la finalidad de que el adquiera seguridad tanto en sus investigaciones, la creatividad y en las implementaciones. 


\section{Conclusión}

Después de la implementación del aprendizaje basado en proyectos vinculado al diseño de experimentos se logró por parte de los estudiantes:

a. La replicabilidad y la reproducibilidad de tres proyectos realizados por los integrantes del grupo:

- Eficiencia de composta \& biocomposta.

- Optimización de celdas de hidrógeno.

- Eficiencia de los biodigestores.

b. La optimización del proceso realizado permite identificar las variables que deben controlarse en la elaboración de cada uno de los productos.

c. Determinación de las instancias que apoyan el desarrollo de proyectos de energías renovables a nivel estatal y nacional.

d. Tres cuartas partes de los egresados se encuentran realizando sus estadías en empresas relacionadas con su carrera.

e. Desarrollo de la creatividad por parte de cada equipo en la implementación de su prototipo y en las innovaciones para mejorar su eficiencia, después de las primeras mediciones.

f. Aplicación adecuada de trabajo colaborativo por parte de cada uno de los equipos, asumiendo los roles de liderazgo en los grupos.

g. Se logró una excelente y efectiva comunicación en un entorno interdisciplinario, por parte de los integrantes de cada equipo.

h. Los estudiantes comprendieron y aplicaron su espíritu empresarial describiendo y fundamentando su plan de negocios.

i. El Diseño de Experimentos permitió identificar más rápidamente tanto las problemáticas como las soluciones fundamentadas promoviendo con esto la toma de decisiones efectivas.

Como puede observarse, los puntos antes mencionados, corresponden a las características que evalúan el desarrollo emprendedor en el estudiante de ingeniería. 11

Como perspectiva a esta implementación se propone dar seguimiento a los egresados a partir del siguiente cuatrimestre en que el periodo de estadías haya concluido.

\section{Referencias}

[1] Ortegón Alvar, I.; Cardoso Landa, G.; importancia del emprendimiento en la formación integral del ingeniero en México; Revista electrónica ANFEI, Digital; año2, no. 5, jun - Dic 2016, issn 2395-9878

[2] Nichols, Steven; E. Armstrong, Neal. (2003). Engineering Entrepreneurship: Does entrepreneurship have a role in engineering education. USA: Antennas and Propagation Magazine, IEEE.

[3] Valencia, A., Carilo, O. \& Aedo, J. (2012). Las tendencias en la ingeniería. Facultad de Ingeniería. Universidad de Antioquia. Ingeniería y Sociedad, 4, PP. 29-31

[4] Mayorga Fernández Dolores Madrid Vivar. (2010). Modelos didácticos y Estrategias de enseñanza en el Espacio Europeo de Educación Superior. Dialnet, 1, 1-21.

[5] Medina Rivilla, Sánchez Romero. (2003). La Innovación en el aula: referente para el diseño y desarrollo curricular. Dialnet, Vol.50.nº $1,62-86$.

[6] Ausubel, d.p.; Novak, j.d. y Hanesian, h. (1983). psicología educativa: un punto de vista cognoscitivo. México, Editorial trillas. traducción al español, de Mario Sandoval p., de la segunda edición de educational psychology: a cognitive view

[7] Enkvist I. (2011). La buena y la mala educación. S: L: Encuentro.

[8] Ojeda Margarita Caballero Edgar Morales Norma V. Galeana. (2012). Hacia una nueva pedagogía: el enfoque basado en proyectos para mejorar el aprendizaje del diseño estadístico. Veracruz, México: Laboratorio de Investigación y Asesoría Estadística (LINAE)

[9] Graham, A. (1987). Statistical investigations in the secondary school. Cambri Open University Centre for Mathematics Education.

[10] Zidek, L. (2010). Engineering Service Learning, Engineering Entrepreneurship and assessment: Building a program that works. Frontiers in Education Conference (FIE), 2010 IEEE (págs. T2D-1). 